\title{
超临界甲醇中 $\mathrm{Ce} / \mathrm{Cu} / \mathrm{Zn} / \mathrm{Al}$ 催化木质素制备酚类 化合物的研究
}

\author{
黄 钰 ${ }^{1,3}$, 包桂蓉 $2,3 *$, 罗 嘉 ${ }^{4}$, 杨智翔 2,3 , 刘 果 ${ }^{2,3}$, 惠 硕 ${ }^{2,3}$, 陈 靖 ${ }^{2,3}$, 李佳蔚 2,3 \\ (1. 昆明理工大学 化学工程学院, 云南昆明 650000 ; \\ 2. 昆明理工大学治金与能源工程学院, 云南昆明 650000 ;
}

3. 昆明理工大学 省部共建复杂有色金属资源清洁利用国家重点实验室, 云南 昆明 650000 ;

4. 中国科学院 西双版纳热带植物园, 云南 昆明 650000)

\begin{abstract}
摘要: 采用共沉淀法制备 $\mathrm{Ce} / \mathrm{Cu} / \mathrm{Zn} / \mathrm{Al}$ 催化剂, 并将其应用于超临界甲醇中木质素的催化液化. 考察单因素条件 对木质素转化率和生成酚类的影响, 得到最佳反应条件: 反应温度 $320{ }^{\circ} \mathrm{C}$, 反应时间 $120 \mathrm{~min}$, 催化剂用量 $150 \mathrm{mg}$, 初始压力 $2 \mathrm{MPa}$, 木质素的转化率达到 $67.33 \%$, 酚类产率达到 $30.84 \%$. 同时, 以苯酚及愈创木酚为模型 物, 比较有无催化剂对液化产物分布或选择性生成的影响. $\mathrm{Ce} / \mathrm{Cu} / \mathrm{Zn} / \mathrm{Al}$ 催化剂的加入促进了甲醇的重整及其与 模型化合物苯酚、愈创木酚的烷基化反应, 从而生成了大量的烷基苯酚.
\end{abstract}

关键词: 木质素; 催化液化; $\mathrm{Cu} / \mathrm{Zn} / \mathrm{Al}$ 催化剂; 苯酚; 超临界甲醇

中图分类号: 0643.32

文献标志码: A

随着化石能源的枯竭以及所带来的环境问题， 寻找新型替代能源刻不容缓. 生物质作为一种清洁 能源、可用资源丰富的能源受到越来越多关注 ${ }^{[1-2]}$. 木质纤维素生物质在自然界蕴藏丰富. 作为木质纤 维素所含的主要成分之一, 木质素可以用作重要的 化工原料, 但因其复杂的化学结构和反应惰性增加 了利用难度. 因此, 如何实现木质素的高效液化成 为研究热点.

催化液化是生物质资源高效利用的主要方式之 一, 是在催化剂和溶剂的共同作用下将大分子降解 为小分子化合物的反应 ${ }^{[3]}$. 超临界流体, 例如超临 界甲醇和乙醇, 具有高介电常数和弱氢键, 且临界 温度和压力低 ${ }^{[4-5]}$, 因此通常用作生物质液化过程 中的溶剂. 在超临界条件下, 醇可直接与木质素发 生反应. Joachim B 等 ${ }^{\left[{ }^{[}\right]}$提出了一种新䓉、简单的非 催化方法, 通过乙醇溶液直接分解木质素产生庚烷 可溶性生物油. Luo 等 ${ }^{[7]}$ 利用碱木质素在超临界乙 醇中的碳化反应生产富碳元素的固体生物炭, 所得 液体产物主要为带一至两个乙基的苯酚, 说明醇在
超临界条件下会参与酚类分子的烷基化反应. 同 时, 甲醇还可以通过重整产生氢气, 为木质素氢解 提供氢源. Galebach 等 ${ }^{[8]}$ 深人研究了纤维素在超临 界甲醇中的解聚和加氢脱氧的反应途径, 证明了 $30 \%$ 的氢是由甲醇重整产生并掺人最终的液体产 物中.

$\mathrm{Cu}$ 基催化剂因高活性、高选择性、低价格被广 泛应用于加氢、脱氧等领域 ${ }^{[9-10]}$. 将 $\mathrm{Cu}$ 基催化剂用 于催化生物质在超临界甲醇中的转化, 能起到催化 生物质和甲醇重整制氢的双功能. Barta 等 ${ }^{[1]}$ 研究 了在掺杂铜的多孔金属氧化物催化剂和超临界甲醇 的作用下催化分解木质素, 从甲醇到有机溶剂木质 素的氢转移导致苯醚键的完全氢解, 以及芳环的氢 化. Wu 等 ${ }^{[12]}$ 制备了双功能 $\mathrm{CuO}-\mathrm{MO} / \mathrm{Al}_{2} \mathrm{O}_{3}(\mathrm{M}=$ $\mathrm{Ce}, \mathrm{Mg}, \mathrm{Mn}, \mathrm{Ni}, \mathrm{Zn}$ ) 催化剂, 并在超临界甲醇中 将纤维素氢解为 $\mathrm{C} 4-\mathrm{C} 7$ 醇. $\mathrm{CuO}$ 基催化剂有促进甲 醇重整生成 $\mathrm{H}_{2}$, 随后发生了纤维素氢解和中间产 物加氢的双重作用, 而助剂如 $\mathrm{CeO}$ 的添加可以提高 C4-C7 醇的产率. $\mathrm{CeO}_{2}$ 的掺人通过减小铜的粒径改

收稿日期: 2020-02-01; 修回日期: 2020-02-12.

基金项目: 国家自然科学基金(51266003) (The National Natural Science Foundation of China [ Grant Number: 51266003]).

作者简介: 黄钰 (1995-), 女, 硕士研究生, E-mail:398236476@ qq.com( Huang Yu(1995-), female, Master candidate, E-mail:398236476@ qq. com).

* 通讯联系人, 包桂蓉, 博士, 博士生导师, E-mail：1633940830@ qq.com. 
善了铜的分散性, 表现出最高的甲醇转化率和生产 $\mathrm{H}_{2}$ 的选择性 ${ }^{[13]}$. 然而, 到目前为止, Ce 改性的 $\mathrm{Cu} /$ $\mathrm{Zn} / \mathrm{Al}$ 催化剂应用到超临界甲醇中催化液化木质素 的报道还很少.

使用 $\mathrm{Ce}$ 改性的 $\mathrm{Cu} / \mathrm{Zn} / \mathrm{Al}$ 催化剂在超临界甲 醇中对木质素进行催化液化实验研究, 考察反应温 度、反应时间、催化剂用量和初始压力对木质素转 化率和生成酚类的影响, 找到最佳反应条件, 探讨 碱木质素在该催化剂作用下转化为烷基酚以及相关 的产物分布. 同时以苯酚和愈创木酚作为模型物, 比较催化剂对液化产物分布或选择性生成的影响.

\section{1 实验部分}

\section{1 实验原料和仪器}

实验所用碱木质素购自东京化成工业株式会社, 碱木质素组成含 $\mathrm{C} 、 \mathrm{H} 、 \mathrm{O} 、 \mathrm{~N} 、 \mathrm{~S}$ 元素 ${ }^{[14]}$, 模型化合 物苯酚、愈创木酚均为分析纯. 硝酸铜 $\left(\mathrm{Cu}\left(\mathrm{NO}_{3}\right)_{2}\right.$. $\left.3 \mathrm{H}_{2} \mathrm{O}\right)$ 、硝酸锌 $\left(\mathrm{Zn}\left(\mathrm{NO}_{3}\right)_{2} \cdot 6 \mathrm{H}_{2} \mathrm{O}\right)$ 、硝酸铝 $\left(\mathrm{Al}\left(\mathrm{NO}_{3}\right)_{3} \cdot 9 \mathrm{H}_{2} \mathrm{O}\right)$ 、硝酸铈 $\left(\mathrm{Ce}\left(\mathrm{NO}_{3}\right)_{3} \cdot 6 \mathrm{H}_{2} \mathrm{O}\right)$ 均为分析纯. 无水碳酸钠、甲醇也均为分析纯. 实验 仪器有美国 Parr 公司 4590 型高温高压反应釜、瑞 土 BUCHI-R215 旋转蒸发仪和 $101 \mathrm{~A}-1$ 电热鼓风干 燥箱等.

\section{2 实验方法}

1.2.1 催化剂制备采用共沉淀法制备 $\mathrm{Ce} / \mathrm{Cu} /$ $\mathrm{Zn} / \mathrm{Al}$ 催化剂. 按一定质量称取 $\mathrm{Cu}\left(\mathrm{NO}_{3}\right)_{2} \cdot 3 \mathrm{H}_{2} \mathrm{O}$ 、 $\mathrm{Zn}\left(\mathrm{NO}_{3}\right)_{2} \cdot 6 \mathrm{H}_{2} \mathrm{O} 、 \mathrm{Al}\left(\mathrm{NO}_{3}\right)_{3} \cdot 9 \mathrm{H}_{2} \mathrm{O} 、 \mathrm{Ce}\left(\mathrm{NO}_{3}\right)_{3} \cdot$ $6 \mathrm{H}_{2} \mathrm{O}$, 依次加人去离子水中 $(\mathrm{Ce} / \mathrm{Cu} / \mathrm{Zn} / \mathrm{Al}$ 摩尔比 为 $1.2: 4.8: 1.9: 0.1)$, 配制成混合盐溶液, 与 $\mathrm{Na}_{2} \mathrm{CO}_{3}$ 水溶液分别置于分液漏斗中. 开启分液漏斗 阀门, 让混合盐溶液与 $\mathrm{Na}_{2} \mathrm{CO}_{3}$ 水溶液并流滴定人 同一烧杯中, 在滴定过程中 $\mathrm{pH}$ 控制在 $8 \sim 8.5$, 水浴 锅温度 $60{ }^{\circ} \mathrm{C}$, 滴定完毕后, 保持 $60{ }^{\circ} \mathrm{C}$ 静置老化 $2 \mathrm{~h}$, 然后多次抽滤洗涤至中性, 在 $105^{\circ} \mathrm{C}$ 干燥箱中 干燥 $12 \mathrm{~h}$, 后放人 $500{ }^{\circ} \mathrm{C}$ 马弗炉中焙烧 $5 \mathrm{~h}$, 冷却后 得到 $\mathrm{Ce} / \mathrm{Cu} / \mathrm{Zn} / \mathrm{Al}$ 催化剂.

1.2.2 碱木质素液化实验方法将木质素、Ce/ $\mathrm{Cu} / \mathrm{Zn} / \mathrm{Al}$ 催化剂按质量比 1:2 与 $15 \mathrm{~mL}$ 甲醇混 合, 置于 $50 \mathrm{~mL}$ 高压反应釜中, 密封, 并用氮气置 换 3 次直至空气排尽, 再充人压力为 $2 \mathrm{MPa}$ 的氮 气, 设置反应温度为 $260 \sim 340{ }^{\circ} \mathrm{C}$, 待温度升到设定 之后开始计时, 反应 $30 \sim 150 \mathrm{~min}$, 实验过程中压力 范围 8.6 20 MPa, 反应结束后自然冷却至室温. 收
集反应后得到的固体残渣和液体产物, 其中液体产 物使用旋转蒸发仪去除溶剂; 固体残渣在 $80{ }^{\circ} \mathrm{C}$ 真 空干燥箱中干燥后并称重.

木质素转化率计算公式见式(1)

$$
X=\frac{m_{0}-m_{1}}{m_{0}} \times 100 \%
$$

式中, $X$ 一木质素转化率, $\% ; m_{0}$ 一木质素质量, $\mathrm{mg} ; m_{1}$ 一固体残渣质量, $\mathrm{mg}$.

1.2.3 苯酚及愈创木酚反应实验苯酚的超临界 反应实验步骤与碱木质素液化实验步骤相似, 设置 反应温度 $320{ }^{\circ} \mathrm{C}$ 、搅拌转速 $300 \mathrm{r} / \mathrm{min}$, 初始压力 2 $\mathrm{MPa}$, 反应 $2 \mathrm{~h}$, 实验过程中, 压力达到 $16 \mathrm{MPa}$ 左 右, 反应结束后自然冷却至室温. 抽滤得到固体残 渣和液体产物, 固体残渣在 $80{ }^{\circ} \mathrm{C}$ 真空干燥箱中干 燥后并称重, 液体产物使用旋蒸仪去除溶剂. 愈创 木酚反应实验的操作及反应条件与苯酚相同.

1.2 .4 液化产物的分析表征 FT-IR 分析选用美 国赛默飞 Nicolet is10 型红外光谱仪, 样品处理采用 溴化钾压片法, 波数范围 $400 \sim 4000 \mathrm{~cm}^{-1}$. GC-MS 分析采用美国 Technologies 公司 HP6890GC/ 5973MS 气相色谱质谱联用仪. GC 条件: HP-5MS; 柱温: $50 \sim 250{ }^{\circ} \mathrm{C}$, 程序升温 $5^{\circ} \mathrm{C} / \mathrm{min}$; 柱流量 1.0 $\mathrm{mL} / \mathrm{min}$; 进样口温度 $250{ }^{\circ} \mathrm{C}$; 柱前压 $100 \mathrm{kPa}$; 进 样量 $1.5 \mu \mathrm{L}$; 分流比 $10: 1$; 载气为高纯氮气. MS 条件: 电离方式 $\mathrm{El}$; 电子能量 $70 \mathrm{eV}$; 传输线温度 $250{ }^{\circ} \mathrm{C}$; 离子源温度 $240{ }^{\circ} \mathrm{C}$; 四极杆温度 $150{ }^{\circ} \mathrm{C}$; 采 用仪器自带的 NIST11 谱库定性判断离子峰信号的 归属. 检测出来的产物采用峰面积归一化法确定各 成分的相对含量.

\section{2 结果与讨论}

\section{1 催化剂表征}

对 $\mathrm{Ce} / \mathrm{Cu} / \mathrm{Zn} / \mathrm{Al}$ 催化剂进行 ICP-OES、XRD、 BET 等表征, 相关表征数据已在研究组之前的工 作 ${ }^{[15]}$ 中列出. 用 ICP-OES 测定催化剂的元素组成, $\mathrm{Ce} / \mathrm{Cu} / \mathrm{Zn} / \mathrm{Al}$ 的摩尔比为 $1.6: 6.7: 2.5: 0.1$, 接 近理论值. 催化剂的 XRD 谱未检测到 $\mathrm{CeO}_{2}$ 的峰, 这意味着 $\mathrm{CeO}_{2}$ 在催化剂载体表面上的分散良好. 与 未添加 $\mathrm{Ce}$ 的 $\mathrm{Cu} / \mathrm{Zn} / \mathrm{Al}$ 催化剂相比, Ce 修饰的 $\mathrm{CuO}$ 和 $\mathrm{ZnO}$ 的 XRD 峰变宽, 相对峰强度变弱. 这表 明添加 $\mathrm{Ce}$ 可能会抑制 $\mathrm{CuO}$ 和 $\mathrm{ZnO}$ 晶体的生长, 这 可能会改善催化剂中活性相的分散性, 并增强这些 活性成分之间的相互作用. 与未添加 Ce 的催化剂 
比, $\mathrm{Ce} / \mathrm{Cu} / \mathrm{Zn} / \mathrm{Al}$ 催化剂的 BET 表面积从 77.6 增 加到 $88.6 \mathrm{~m}^{2} \cdot \mathrm{g}^{-1}$, 孔体积从 0.218 增大到 0.265 $\mathrm{cm}^{3} \cdot \mathrm{g}^{-1}$, 平均孔径从 6.45 增加到 $9.01 \mathrm{~nm}$, 表明 Ce 的加人能增大催化剂的 BET 表面积.

\section{2 反应条件对液体产物的影响}

\subsection{1 反应温度的影响在木质素 $300 \mathrm{mg}$ 、甲醇} $15 \mathrm{~mL}$ 、催化剂 $150 \mathrm{mg}$ 和反应时间 $120 \mathrm{~min}$ 的条件 下, 考察了反应温度对木质素转化率及液相产物产 率的影响. 表 1 是不同反应温度下 GC-MS 检测到的
液体产物的主要组成成分, 有酚类化合物和醚类化 合物，如 4,5-二甲基间苯二酚、2,4,6-三甲基苯酚、 邻苯二甲醚等. 由图 1 可知, 随着温度的升高, 木 质素的转化率随之升高, 在 $340{ }^{\circ} \mathrm{C}$ 时达到 $68 \%$. 酚 类产率随着温度的升高先增加, 在 $320{ }^{\circ} \mathrm{C}$ 时达到 $30.84 \%$, 当温度高于 $320^{\circ} \mathrm{C}$ 后, 酚类产率反而下降. 由表 1 可以看出, 随着温度的升高, 醚类化合物和 三甲基苯酚的含量减少，四甲基苯酚、罗甲基苯含 量增加.

\section{表 1 不同反应温度下 $\mathrm{Ce} / \mathrm{Cu} / \mathrm{Zn} / \mathrm{Al}$ 催化木质素的产物分布}

Table 1 Product distribution of lignin catalyzed by $\mathrm{Cu} / \mathrm{Zn} / \mathrm{Al} / \mathrm{Ce}$ at different reaction temperatures

\begin{tabular}{|c|c|c|c|c|c|c|}
\hline \multirow{2}{*}{$\begin{array}{l}\mathrm{RT} \\
/ \mathrm{min}\end{array}$} & \multirow{2}{*}{ Identification } & \multicolumn{5}{|c|}{ Area $\%$} \\
\hline & & $260^{\circ} \mathrm{C}$ & $280{ }^{\circ} \mathrm{C}$ & $300^{\circ} \mathrm{C}$ & $320{ }^{\circ} \mathrm{C}$ & $340{ }^{\circ} \mathrm{C}$ \\
\hline 11.038 & 2, 3, 4-trimethyl-2-cyclopentenone & 2.632 & 2.453 & 1.17 & 1.165 & 1.12 \\
\hline 12.373 & 2, 3,4,5-tetramethyl-2-cyclopentenone & 8.53 & 8.455 & 6.474 & 4.429 & 3.253 \\
\hline 13.512 & Veratrole & 2.229 & 1.407 & 0.891 & - & - \\
\hline 13.634 & 4, 5-dimethylresorcinol & 2.059 & 2.118 & 1.482 & 1.422 & - \\
\hline 13.917 & 2, 3,5,6-tetramethyl-2-cyclopentenone & - & 2.008 & 1.431 & 1.273 & 1.125 \\
\hline 15.151 & 2, 4, 6-trimethyltoluene & 6.533 & 6.074 & 5.733 & 4.195 & 2.915 \\
\hline 16.159 & 3, 4-dimethoxytoluene & 2.93 & 2.172 & 1.527 & 0.693 & - \\
\hline 17.105 & tert-Butylhydroquinone & - & 1.145 & 1.160 & - & - \\
\hline 17.256 & Trimethylhydroquinone & 1.419 & - & - & - & - \\
\hline 17.307 & Pentamethylbenzene & 1.13 & 1.84 & 2.43 & 2.947 & 2.732 \\
\hline 18.986 & 5-methoxy-2, 3, 4trimethylphenol & 5.194 & 4.056 & - & - & - \\
\hline 19.384 & $2,3,4,6$-tetracresol & 3.549 & 4.789 & 5.47 & 7.169 & 7.647 \\
\hline 19.425 & $2,3,5,6$-tetracresol & 7.86 & 7.722 & 9.519 & 11.53 & 11.689 \\
\hline 21.425 & Butylbenzylalcohol & 2.391 & 2.71 & 3.24 & 3.875 & 3.54 \\
\hline 22.328 & 2-tert-Butyl-4,6-dimethylphenol & 1.708 & 2.118 & 3.012 & 4.962 & 4.215 \\
\hline 23.013 & 3-tert-butyl-4-hydroxyanisole & 12.038 & 12.725 & 5.77 & 3.249 & - \\
\hline 23.497 & 1,3-cyclopentadiene-5-dimethyl-1, 2-dipropyl & - & 0.724 & 0.671 & 1.151 & 0.982 \\
\hline 23.707 & 1-methoxy-4-methyl-2-propan-2-ylbenzene & 9.16 & 10.173 & 12.165 & 13.013 & 13.987 \\
\hline 24.818 & 6-tert-butyl-2,4-xylenol & 0.924 & 0.824 & 1.243 & 1.011 & 0.915 \\
\hline 25.144 & Diprivan & 2.312 & 2.393 & 2.464 & 2.838 & 3.156 \\
\hline 27.307 & 2-ethyl-5, 7-dimethyl-1-benzothiophen & 0.671 & 0.834 & 1.124 & 1.011 & 1.241 \\
\hline 27.117 & 1,2-diethyl-3,4,5,6-tetramethylbenzen & 0.873 & 1.103 & 1.134 & 0.985 & 1.21 \\
\hline 28.992 & 5,6,7,8-tetramethyl-1,2,3,4-tetrahydronaphthalene & 0.972 & 0.647 & 0.876 & 1.163 & 1.215 \\
\hline
\end{tabular}




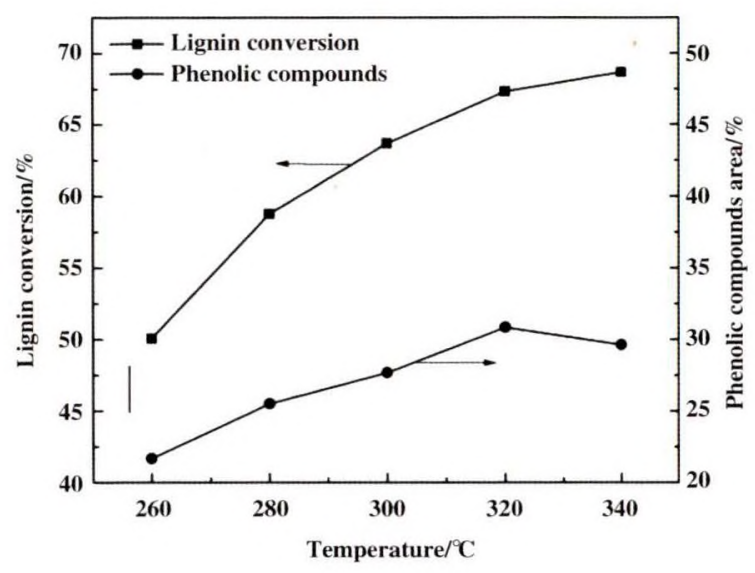

图 1 反应温度对木质素转化率及酚类产率的影响

Fig. 1 Effects of reaction temperature on the lignin conversion and the yield of phenolics

\subsection{2 反应时间的影响在木质素 $300 \mathrm{mg}$ 、甲醇} $15 \mathrm{~mL}$ 、催化剂 $150 \mathrm{mg}$ 和反应温度 $320^{\circ} \mathrm{C}$ 的条件 下，考察反应时间对木质素转化率及酚类产率的影 响. 由图 2 可知, 木质素转化率随反应时间增加而

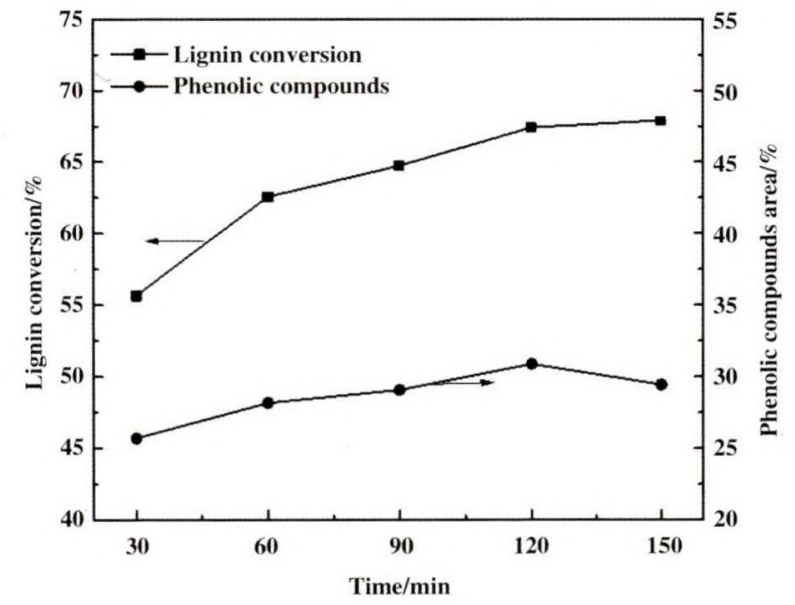

图 2 反应时间对木质素转化率及酚类产率的影响

Fig.2 Effects of reaction time on the lignin conversion and the yield of phenolics

呈上升趋势, 在 30 60 min 反应时间内上升较快, 在 $150 \mathrm{~min}$ 时达到 $67.33 \%$. 随反应时间的增加酚类 产率先增后减, 但总体来说变化不大, 说明延长反 应时间对酚类产率的提升不大.

\section{2 .3 催化剂用量在木质素 $300 \mathrm{mg}$ 、甲醇 15} $\mathrm{mL}$ 、反应时间 $120 \mathrm{~min}$ 和反应温度 $320{ }^{\circ} \mathrm{C}$ 的条件 下, 考察催化剂用量对木质素转化率及酚类产率的 影响. 随着催化剂用量的增加, 木质素转化率和酚
类产率都是呈先升后降的趋势, 如图 3 所示. 当催 化剂用量为 $150 \mathrm{mg}$ 时, 木质素转化率达到最大值

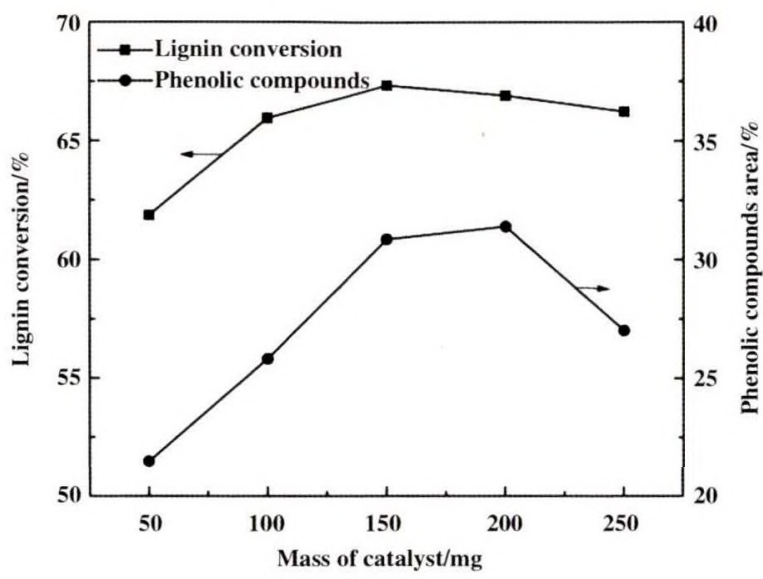

图 3 催化剂用量对木质素转化率及酚类产率的影响

Fig. 3 Effects of dosage of catalyst on the lignin conversion and the yield of phenolics

为 $67.33 \%$. 而催化剂用量的继续增加, 木质素的转 化率开始呈下降趋势, 这可能是因为过多催化剂的 加人使催化剂发生聚集并沉降，从而导致催化剂与 反应物接触面积减少, 催化效果减弱, 木质素转化 率下降. 而酚类产物活性高，在过多催化剂的作用 下，彼此间更易于进行缩聚反应，因此酚类产物的 产率降低 ${ }^{[16]}$.

2.2.4 初始压力的影响 由图 4 可知, 初始压力 越大对木质素的降解越有利. 木质素转化率随初始

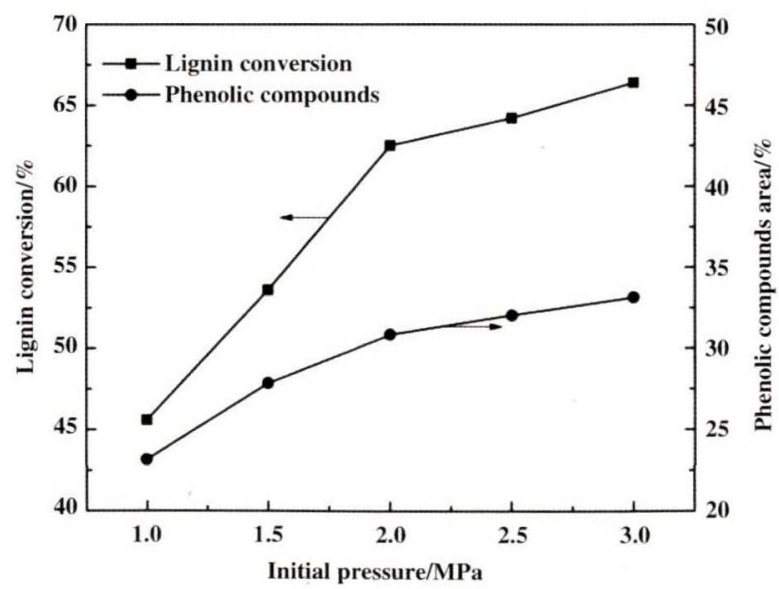

图 4 不同初始压力对木质素转化率的影响

Fig. 4 Effects of initial pressure on the lignin conversion and the yield of phenolics

压力的增大而增加, 初始压力为 $1 \mathrm{MPa}$ 时木质素转 化率仅为 $45.6 \%$, 当初始压力为 $3 \mathrm{MPa}$ 时可以达到 
$66.4 \%$. 随着初始压力继续升高木质素转化率提高 不大, 但是过高的压力会导致设备使用寿命的缩 短, 因此选择适合的初始压力为 $2 \mathrm{MPa}$.

\section{3 产物的 FT-IR 分析}

木质素、液化产物及固体残渣的 FT-IR 分析如 图 5 所示. 从图中可以看出, 在 $3450 \mathrm{~cm}^{-1}$ 附近存在

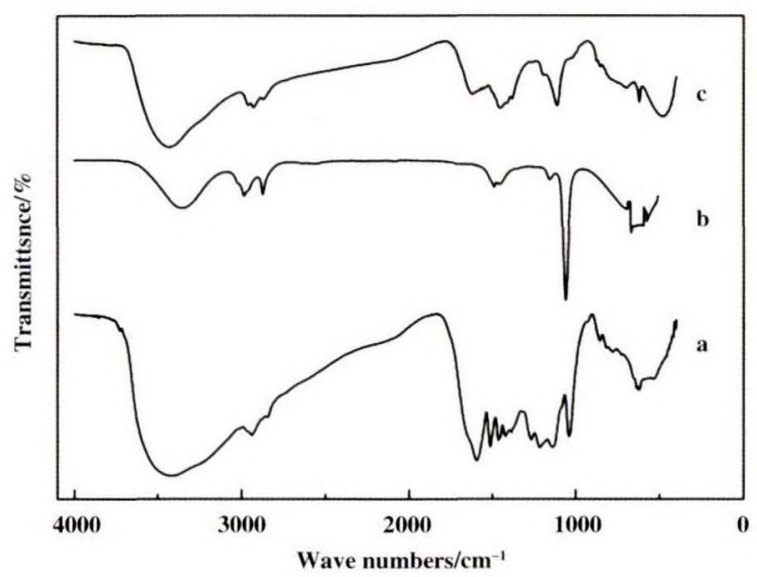

图 5 木质素 (a), 液化产物 (b) 及固体残渣 (c) 的 FI-IR 分析

Fig.5 FI-IR analysis of lignin (a), liquefaction product (b) and residue (c)

$\mathrm{O}-\mathrm{H}$ 的伸缩振动峰, $1700 \mathrm{~cm}^{-1}$ 附近存在 $\mathrm{C}=\mathrm{O}$ 的 伸缩振动峰 ${ }^{[17]}$, 在 $1505 \sim 1605 \mathrm{~cm}^{-1}$ 区域内的峰是 芳香环化合物的骨架振动 ${ }^{[18]}$. 在 1200 1300、1324 $\mathrm{cm}^{-1}$ 附近存在 $\mathrm{C}-\mathrm{O}$ 和 $\mathrm{C}-\mathrm{O}-\mathrm{C}$ 的振动吸收

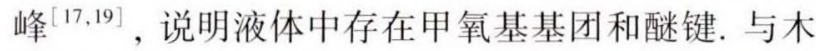
质素相比, 液体产物在 1200 1300、1324 $\mathrm{cm}^{-1}$ 附近 存在 $\mathrm{C}-\mathrm{O}$ 和 $\mathrm{C}-\mathrm{O}-\mathrm{C}$ 的振动吸收峰减弱, 说明液 化过程中醚键大量断裂.

\section{4 超临界甲醇中木质素催化解聚机理探究}

以苯酚和愈创木酚作为模型物, 分析催化剂对 比于无催化条件下对液化产物分布或选择性生成的 影响. 苯酚在超临界甲醇中的液化产物 GC-MS 总离 子流图如图 6 所示. 从图中可以看出, 在不加催化 剂的情况下, 苯酚只能与少量甲醇反应生成 2-甲基 苯酚. 在加人催化剂后液化产物主要组分有间二甲 苯、1,3,5-三甲基苯、2,4,6-三甲基苯酚、2,6-二甲 基醚等, 表明 $\mathrm{Ce} / \mathrm{Cu} / \mathrm{Zn} / \mathrm{Al}$ 催化剂的加人促进了苯 酚与甲醇发生烷基化反应.

图 7 为愈创木酚降解产物的总离子流图. 在不 加催化剂, 反应时间 $4 \mathrm{~h}$ 条件下, 愈创木酚中的甲 氧基键没有断裂，只有少量愈创木酚反应得到甲基 愈创木酚. 加人催化剂后，愈创木酚的烷氧基团发

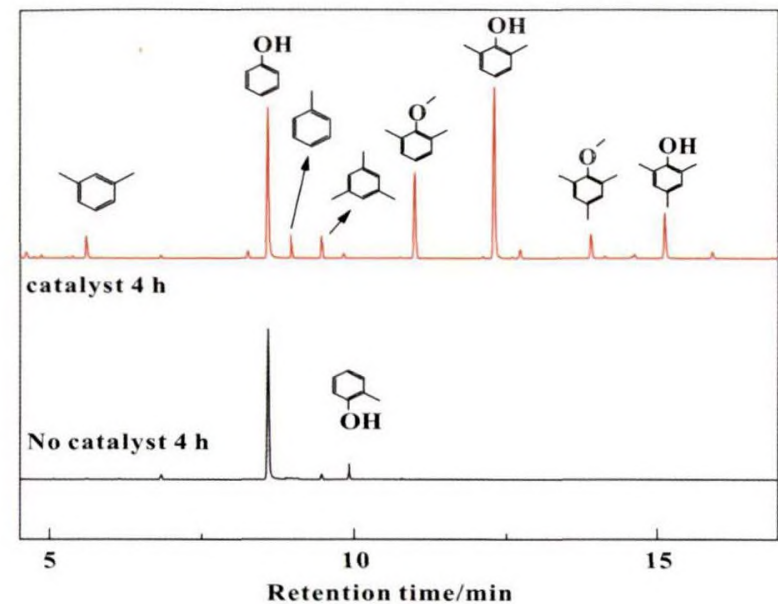

图 6 苯酚在超临界甲醇中降解产物的 GC-MS 总离子流图

Fig.6 GC-MS total ion chromatograms of degradation products of phenol in supercritical methanol

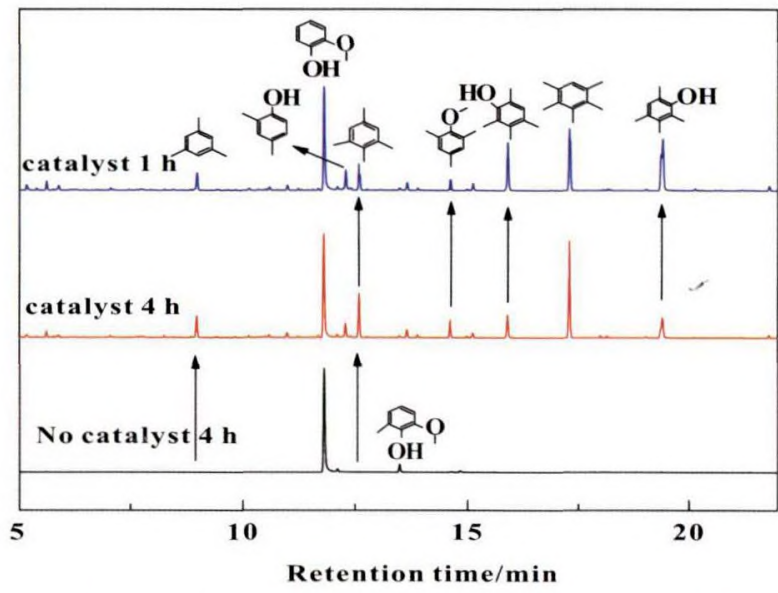

图 7 愈创木酚在超临界甲醇中降解产物的 GC-MS 总离子流图

Fig.7 GC-MS total ion chromatograms of degradation products of guaiacol in supercritical methanol

生断裂, 形成烷基苯酚, 随着反应时间的增加, 烷 基苯酚发生烷基反应形成多甲基苯. 液化产物主要 组分有 2,4,6-三甲基苯酚、1,3,4,5-三甲基苯酚、 2,4-二甲基苯酚、1,3,5-三甲基苯、1,3,4,5-四甲基 苯等.

\section{3 结论}

超临界甲醇中以 $\mathrm{Ce}$ 改性 $\mathrm{Cu} / \mathrm{Zn} / \mathrm{Al}$ 为催化剂 降解木质素制备酚类, 在单因素实验中, 得到最佳 条件为: 反应温度 $320^{\circ} \mathrm{C}$, 反应时间 $120 \mathrm{~min}$, 催化 剂用量 $150 \mathrm{mg}$, 初始压力 $2 \mathrm{MPa}$, 此时木质素的转 化率达到 $67.33 \%$, 酚类产率达到 $30.84 \%$. 随着温 
度的升高, 液化产物中三甲基苯酚含量减少, 而四 甲基苯酚含量增加. 以苯酚及愈创木酚为模型物, 比较有无催化剂对液化产物分布或选择性生成的影 响. 在无催化条件下, 仅与甲醇反应生成带一个甲 基的苯酚和愈创木酚, 而加人 $\mathrm{Ce} / \mathrm{Cu} / \mathrm{Zn} / \mathrm{Al}$ 催化剂 后, 加快了甲醇与模型物的烷基化反应, 产生多甲 基苯酚和多甲基苯. Ce 改性的 $\mathrm{Cu} / \mathrm{Zn} / \mathrm{Al}$ 催化剂促 进了甲醇的重整及其与模型化合物苯酚、愈创木酚 的烷基化反应, 从而生成了大量的烷基苯酚.

\section{参考文献:}

[1] Kubilay Tekin, Selhan Karagöz, Sema Bektaş. A review of hydrothermal biomass processing $[\mathrm{J}]$. Renew Susta En Rev, 2014, 40: 673-687.

[2] Vineet Singh Sikarwar, Ming Zhao, Peter Clough, et al. An overview of advances in biomass gasification $[\mathrm{J}]$. En Environ Sci, 2016, 9(10): 2939-2977.

[3] Luo Xian-feng (罗显峰), Jin Qing-hui (金庆辉), Sun Xiong-kang (孙雄康). Research progress on catalysts applied in catalytic liquefaction of lignin (木质素催化液 化用催化剂的研究进展) [J]. Guangzhou Chem Indus (广州化工), 2017, 45(22) : 4-5.

[4] Wu Xiao-yu(吴晓宇), Lv Xiu-yang(吕秀阳). Chemical reactions in supercritical methanol (超临界甲醇中的化 学反应) [J]. Mod Chem Indus (科技进展), 2009, 29 (12) : 26-30.

[5] Tsukahara T, Harada M, Tomiyasu H, et al. NMR studies on effects of temperature, pressure, and fluorination on structures and dynamics of alcohols in liquid and supercritical states $[\mathrm{J}]$. J Phys Chem A, 2008, 112 (40): 9657-9664.

[6] Nielsen J B, Anders Jensen, Madsen L R, et al. Noncatalytic direct liquefaction of biorefinery lignin by ethanol [J]. En Fuels, 2017, 31(7): 7223-7233.

[7] Luo Jia, Yang Ya-ting, Yang Xing-xia, et al. The formation of char, gaseous and liquid products during lignin carbonization in super- and subcritical solvents $[\mathrm{J}]$. Chem Select, 2017, 2(9): 2828-2831.

[8] Galebach P, Mcclelland D J, Eagan N M et al. Production of alcohols from cellulose by supercritical methanol depolymerization and hydrodeoxygenation $[\mathrm{J}]$. Susta Chem \& Eng, 2018, 6( 8) : 4330-4344.

[9] Turco M, Bagnasco G, Costantino U, et al. Production of hydrogen from oxidative steam reforming of methanol: II. Catalytic activity and reaction mechanism on $\mathrm{Cu} / \mathrm{ZnO} /$
$\mathrm{Al}_{2} \mathrm{O}_{3}$ hydrotalcite-derived catalysts $[\mathrm{J}]$. J Catal, 2004, 228 ( 1$): 56-65$.

[10] Yfanti V L, Vasiliadou E S, Lemonidou A A. Glycerolhydro-deoxygenation aided by in situ $\mathrm{H}_{2}$ generation viamethanol aqueous phase reforming over a $\mathrm{Cu}-\mathrm{ZnO}-\mathrm{Al}_{2} \mathrm{O}_{3}$ catalyst [J]. Catal Sci \& Technol, 2016, 6(14) : 54155426.

[11] Barta K, Matson T D, Fettig M L, et al. Catalytic disassembly of an organosolv lignin via hydrogen transfer from supercritical methanol $[\mathrm{J}]$. Green Chem, 2010, 12(9): 1640-1647.

[12] Wu Yan-hua, Gu Fang-na, Xu Guang-wen, et al. Hydrogenolysis of cellulose to $\mathrm{C} 4-\mathrm{C} 7$ alcohols over bi-functional $\mathrm{CuO}-\mathrm{MO} / \mathrm{Al}_{2} \mathrm{O}_{3}(\mathrm{M}=\mathrm{Ce}, \mathrm{Mg}, \mathrm{Mn}, \mathrm{Ni}, \mathrm{Zn})$ catalysts coupled with methanol reforming reaction $[\mathrm{J}]$. Biores Technol, 2013, 137: 311-317.

[13] Sanjay Patel, Pant K K. Selective production of hydrogen via oxidative steam reforming of methanol using $\mathrm{Cu}-\mathrm{Zn}$ Ce-Al oxide catalysts [J]. Chem Eng Sci, 2007, 62(18/ 20) : 5436-5443.

[14] WangWen-liang, Ren Xue-yong, Li Lu-fei, et al. Catalytic effect of metal chlorides on analytical pyrolysis of alkali lignin $[\mathbf{J}]$. Fuel Proce Technol, 2015, 134: $345-$ 351.

[15] Liu Guo, Bao Gui-rong, Wang Hua, et al. Ce modified $\mathrm{Cu} / \mathrm{Zn} / \mathrm{Al}$ catalysts for direct liquefaction of microcrystalline cellulose in supercritical methanol $[\mathrm{J}]$. Cellulose, 2019, 26: 8291-8300.

[16] Du Fang-li (杜芳黎), Li Yan-ming (黎演明), Xian Xue-quan(洗学权), et al. Lignin to monophenols in ionic liquid-water system( 离子液体-水共溶剂作用下木 质素转化为酚类物质的研究) [ $\mathrm{J}]$. Mod Chem Indus (现代化工) , 2019, 39(2): 144-148.

[17] Shui Heng-fu, Zou De-hai, Wu Hui-hui, et al. Co-liquefaction of xilinguole lignite and lignin in ethanol/water solvents under a cheap iron ore catalyst $[\mathrm{J}]$. Fuel, 2019, 251: 629-635.

[18] Yuan Tong-qi, He Jing, Xu Feng, et al. Fractionation and physico-chemical analysis of degraded lignins from the black liquor of Eucalyptus pellita KP-AQ pulping $[\mathrm{J}]$. Poly Degra Stab, 2009, 94(7): 1142-1150.

[19] Zou De-hai, Yang Xue, Shui Heng-fu, et al. Liquefaction of thermal extracts from co-thermal dissolution of a sub-bituminous coal with lignin and reusability of Ni-Mo$\mathrm{S} / \mathrm{Al}_{2} \mathrm{O}_{3}$ catalyst $[\mathrm{J}]$. J Fuel Chem Technol, 2019, 47 (1) : 23-30. 


\title{
Study on $\mathrm{Ce} / \mathrm{Cu} / \mathrm{Zn} / \mathrm{Al}$ Catalyzed Conversion of Lignin to Phenols in Supercritical Methanol
}

\author{
HUANG Yu ${ }^{1,3}$, BAO Gui-rong ${ }^{2,3 *}$, LUO JIA ${ }^{4}$, YANG Zhi-xiang ${ }^{2,3}$, LIU Guo ${ }^{2,3}$, HUI Shuo ${ }^{2,3}$, \\ CHEN Jing ${ }^{2,3}$, LI Jia-wei ${ }^{2,3}$ \\ (1. Faculty of Chemical Engineering, Kunming University of Science and Technology, Kunming 650000, China; \\ 2. College of Metallurgy and Energy Engineering, Kunming University of Science and Technology, \\ Kunming 650000, China; \\ 3. State Key Laboratory of Complex Nonferrous Metal Resources Clean Utilization, Kunming University of \\ Science and Technology, Kunming 650000, China; \\ 4. Xishuangbanna Tropical Botanical Garden, Chinese Academy of Sciences, Kunming 650000, China)
}

\begin{abstract}
Ce} / \mathrm{Cu} / \mathrm{Zn} / \mathrm{Al}$ catalyst was prepared by co-precipitation method and applied to the catalytic liquefaction of lignin in supercritical methanol. The effects of single conditions on lignin conversion and phenol formation were investigated, and the optimal reaction conditions were obtained: reaction temperature $320{ }^{\circ} \mathrm{C}$, reaction time 120 min, catalyst dosage $150 \mathrm{mg}$, initial pressure $2 \mathrm{MPa}$. The conversion of lignin reached $67.33 \%$, and the yield of phenols reached $30.84 \%$. At the same time, the effects of the presence or absence of catalysts on the types and selectivities of liquefied products were analyzed using phenol and guaiacol as model substances. $\mathrm{Ce} / \mathrm{Cu} / \mathrm{Zn} / \mathrm{Al}$ promoted the reformation of methanol and its alkylation reaction with model compounds phenol and guaiacol, so that a large amount of alkylphenol was formed.
\end{abstract}

Key words : lignin; catalytic liquefaction; $\mathrm{Cu} / \mathrm{Zn} / \mathrm{Al}$ catalyst ; phenols; supercritical methanol 


\section{Study on $\mathrm{Ce} / \mathrm{Cu} / \mathrm{Zn} / \mathrm{Al}$ Catalyzed Conversion of Lignin to}

\section{Phenols in Supercritical Methanol}

HUANG Yu, BAO Gui-rong, LUO JIA, YANG Zhi-xiang,

LIU Guo, HUI Shuo, CHEN Jing, LI Jia-wei

J. Mol. Catal. (China) 2020, 34(1): 058 064

$\mathrm{Ce} / \mathrm{Cu} / \mathrm{Zn} / \mathrm{Al}$ catalyst was prepared by co-precipitation method and was used in the liquefaction of ligninin supercritical methanol. The effect of the presence or absence of catalyst on the distribution or selective formation of liquefied products was compared. The results showed that the $\mathrm{Ce} \operatorname{modified} \mathrm{Cu} / \mathrm{Zn} / \mathrm{Al}$

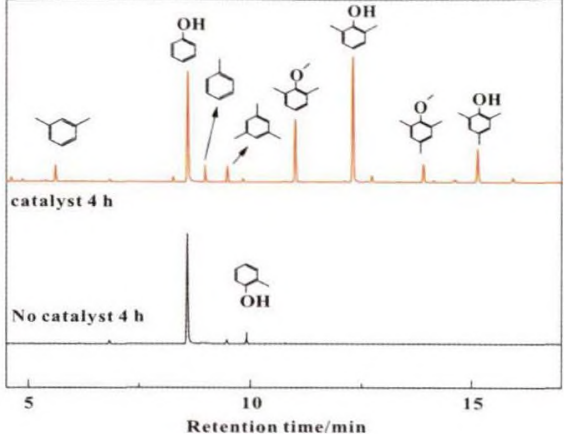

catalysts promoted the reformation of methanol and its alkylation reaction with model compounds phenol and guaiacol, resulting large amount of alkylphenols.

The Research of CO Monoxide in Different Atmosphere on $\mathrm{Au} / \mathrm{Cu} / \mathrm{FLA}$ Catalysts at Low Temperature

SU Hui-juan, ZHENG Yu-hua, SUN Xun, SUN Li-bo,

QI Cai-xia

J. Mol. Catal. (China) 2020, 34(1): 065 071

$\mathrm{Au} / \mathrm{Cu} / \mathrm{FLA}$ series catalysts were prepared by the isovolume method. The relationship between catalyst-structrue and initial activity, dry-feed stable, wet-feed stable stable catalytic
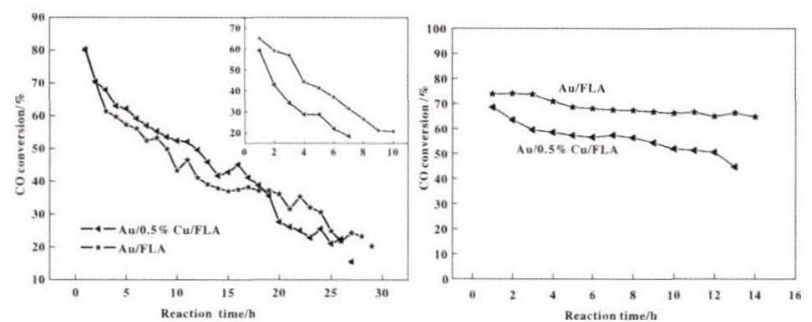

performance of catalysts were investigated by BET, TEM, X-ray diffraction and Infrared spectrum.

\section{Preparation of Pd@s $s$ S-NMe ${ }_{2} /$ AC Nanocatalysts and Their} Application in $\mathrm{C}-\mathrm{C}$ Coupling Reactions

JIANG Jia-lin, JIANG Lei, HOU Zhao-min, GUO Fang

J. Mol. Catal. (China) 2020, 34( 1): 072 080

Pd@sPS-NMe 2 /AC catalysts were prepared by three different methods. The morphology of supporters and immobilization of Pd nanoparticles were characterized by SEM and TEM. The $\mathrm{Pd}$ catalyst on a $s \mathrm{PS}-\mathrm{NMe}_{2} / \mathrm{AC}$ supporter prepared by volatilizing the solvent of a mixed solution of $s \mathrm{PS}-\mathrm{NMe}_{2}$ and AC showed 